Review

\title{
The regulatory networks of the Hippo signaling pathway in cancer development
}

\author{
Maonan Wang ${ }^{1,2}$, Manli Dai ${ }^{3}$, Dan Wang ${ }^{1,2}$, Wei Xiong1,2, Zhaoyang Zeng ${ }^{1,2}{ }^{\circledR}$ and Can Guo ${ }^{1,2}$ \\ 1. NHC Key Laboratory of Carcinogenesis, Hunan Cancer Hospital and the Affiliated Cancer Hospital of Xiangya School of Medicine, Central South \\ University, Changsha, Hunan, China. \\ 2. Key Laboratory of Carcinogenesis and Cancer Invasion of the Chinese Ministry of Education, Cancer Research Institute, Central South University, \\ Changsha, Hunan, China. \\ 3. Hunan Food and Drug Vocational College, Changsha 410036, China. \\ $\bowtie$ Corresponding authors: E-mail: zengzhaoyang@csu.edu.cn (Z.Z.) guocde@csu.edu.cn (C.G).
}

(C) The author(s). This is an open access article distributed under the terms of the Creative Commons Attribution License (https://creativecommons.org/licenses/by/4.0/). See http://ivyspring.com/terms for full terms and conditions.

Received: 2021.05.06; Accepted: 2021.08.15; Published: 2021.08.28

\begin{abstract}
The Hippo signaling pathway is a relatively young tumor-related signaling pathway. Although it was discovered lately, research on it developed rapidly. The Hippo signaling pathway is closely relevant to the occurrence and development of tumors and the maintenance of organ size and other biological processes. This manuscript focuses on YAP, the core molecule of the Hippo signaling pathway, and discussion the upstream and downstream regulatory networks of the Hippo signaling pathway during tumorigenesis and development. It also summarizes the relevant drugs involved in this signaling pathway, which may be helpful to the development of targeted drugs for cancer therapy.
\end{abstract}

Key words: Hippo signaling pathway; YAP; Tumor development

\section{Introduction}

In addition to the classical eight cancer-related signaling pathways, with the development of scientific research, more and more signaling pathways have been found to play a significant role in the development of cancer [1]. The Hippo pathway found in Drosophila is a crucial entry point for understanding the molecular mechanisms that control organ growth during development and regeneration [2-4]. The intracellular environment's inhibitory growth signal activates the Hippo signaling pathway, causing a series of Kinase cascade phosphorylation. MST1/2 (mammalian STE20-like protein kinase) phosphorylation and cofactor SAV1 (human Salvadorhomology 1) promote LATS1/2 (large tumor suppressor1/2) phosphorylation and cofactor MOB1 (MOB kinase activator) of downstream [5-7]. Then, the phosphorylation of downstream effector YAP (Yes-associated protein) /TAZ (Tafazzin) could be increased. Phosphorylated YAP/TAZ could be in the cytoplasm through binding 14-3-3 proteins [8,9] and it could promote proteasome degradation, eventually inhibiting cell proliferation $[7,10,11]$. However, when the cells become cancerous, Hippo pathways are suppressed. Once the "switch" closes, the unphosphorylated YAP/TAZ may enter the nucleus, as a co-transcriptional coactivator [12]. Then, it binds to the TEA domain family members (TEAD) transcription family and promotes the expression of oncogenes associated with tumorigenesis. Finding the switching mechanism related to the Hippo pathway is very necessary for the fight against cancer [13,14]. This review focuses on the upstream and downstream regulatory networks of Hippo pathways, biological effects in tumor formation, and advances in therapeutic cancer targets in pathways.

\section{The role of Hippo signal pathway}

The Hippo signaling pathway was first discovered in Drosophila and had a high evolutionary conservation [15]. In the early stages of cancer, a large number of cancer-derived missense mutations occurred in the highly conserved Hippo pathway, and these mutations caused the Hippo pathway to be inhibited [16]. The kernel components of the Hippo 
pathway include the upstream kinase MST1/2 and LATS1/2, cofactor SAV1, spherical scaffold protein MOB1A/B [17], transcription coactivator YAP and $\mathrm{TAZ}$, and transcription enhancing association domain TEADs. SAV1 is the primary molecule in the Salvador pathway, LATS1/2 is the central molecule in the Warts pathway, and MST1/2 is the central molecule in the Hippo pathway. Therefore, the Hippo signaling pathway can also be recorded as the Salvador/Warts/ Hippo pathway.

When the Hippo pathway is activated, the upstream signal molecule activates MST1/2 and binds to the co-regulatory protein SAV1. Then, phosphorylated LATS1/2 binds to the co-regulatory protein MOB1. After that, the phosphorylation of YAP/TAZ has been activated and binds to the scaffold protein 14-3-3. The YAP/TAZ will be in the cytoplasm and is degraded by the proteasome after ubiquitination. Finally, the biological function of YAP/TAZ is inhibited [18]. When cancer happens, the Hippo pathway is blocked or inactivated, and the phosphorylation cascade is affected. The unphosphorylated YAP/TAZ enters the nucleus from the cytoplasm. It combines with the TEADs transcription family, acting as a transcriptional coactivator, synergistically promoting the expression of target genes, and enhancing cell proliferation and anti-apoptosis [19,20] (Fig. 1).

It is worth noting that the change of MST1/2 does not necessarily cause changes in the Hippo

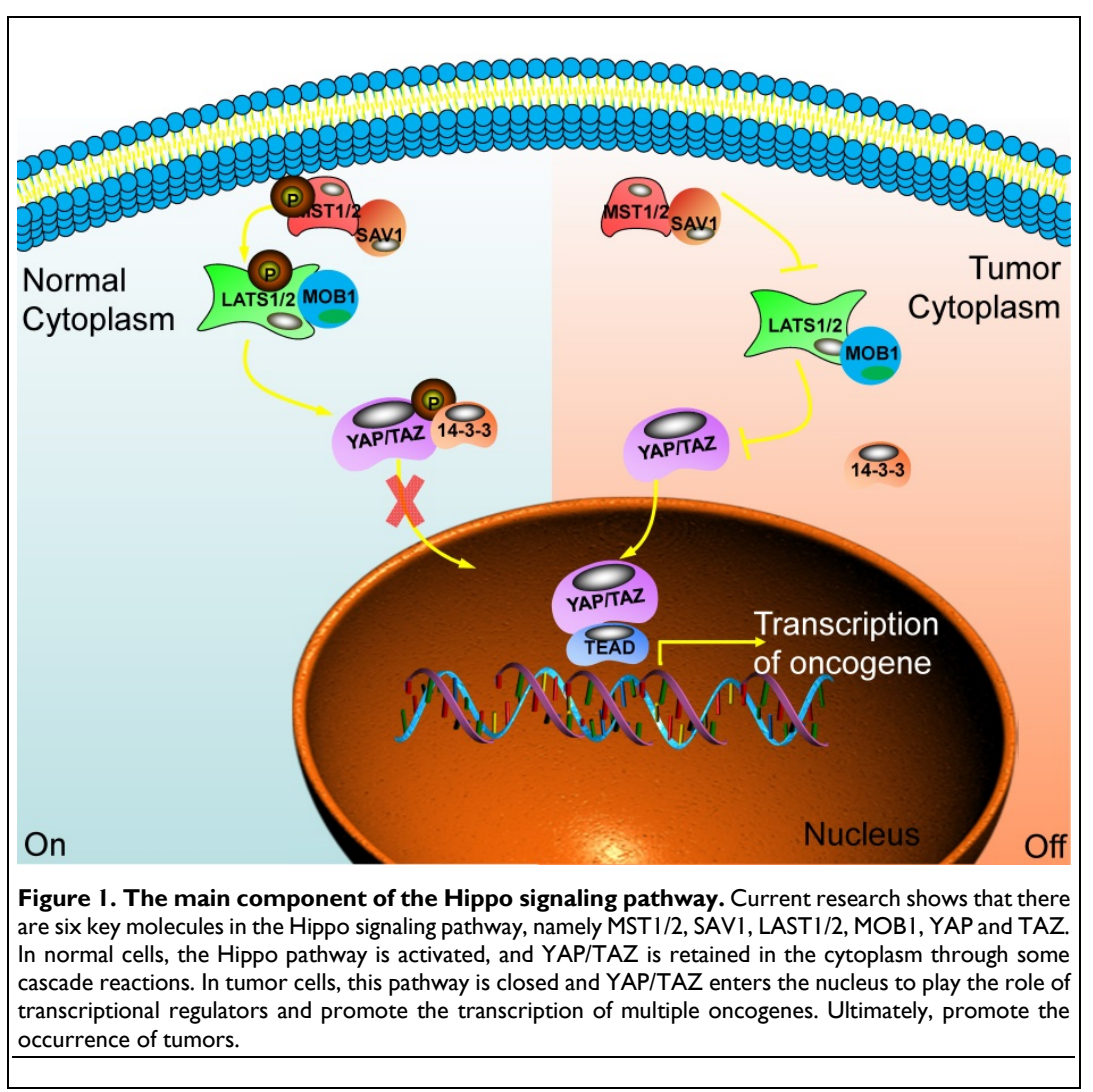

pathway. It could also regulate proteins that do not participate in the Hippo pathway, such as NDR1/2 (Late embryogenesis abundant (LEA) hydroxyproline-rich glycoprotein family), forkhead box O1 (FOXO1)), etc. Similarly, activation of YAP/TAZ does not necessarily require MST1/2. It can also act on LATS1/2 or be directly regulated by other proteins. For example, Hppy and Wsn could inhibit the transcriptional activity of Yki by activating Wts kinase activity. Not all proteins and functions regulated by MST1/2 are therefore definable as Hippo pathways, specifically regulating YAP or LATS1/2 kinase activity only. The protein pathway of /TAZ transcriptional activity is classified as the Hippo signaling pathway [21].

\section{The upstream regulatory networks of the Hippo signaling pathway}

The Hippo signaling pathway is very evolutionarily conserved and is involved in maintaining tissue homeostasis. When the pathway is closed by extracellular signals, it often induces cancer. Abnormal expression of the Hippo pathway has been detected in various cancers, accompanied by changes in extracellular signals. With the deepening of research, more and more upstream regulatory networks of the Hippo signaling pathway have been unearthed [22] (Fig. 2).

\section{Regulation of cell connection on the Hippo signaling pathway}

There are direct or indirect connections between cells in multicellular organisms, and cell connection is an important area where cells are connected. Cell junction related proteins are located on the cell membrane. Mainly divided into tight junctions (TJs) [23] and adhesive connections (AJs) [24]. Angiomotin (AMOT) is complicated in the regulation of tight junctions and tubular formation, migration of vascular endothelial cells, and signal transduction of the Hippo pathway. There are two molecular mechanisms for AMOT to regulate YAP/TAZ activity: one is that AMOT directly binds YAP/TAZ and retains them in the cytoplasm to inhibit its activity expression. This interaction does not rely on Hippo pathway-mediated YAP/TAZ phosphorylation [25]. The other is the upstream kinase that could act on the Hippo pathway. AMOT could directly bind to LATS2 and up-regulate 
the expression of LATS2, ultimately inhibiting the expression activity of YAP/TAZ [26]; it could also stimulate the activation of MST1/2. Then, the phosphorylation of LATS1/ 2 is promoted through the cascade phosphorylation reaction of the Hippo pathway to activate LATS1/2, and finally, the expression activity of YAP/TAZ is inhibited [27]. Besides, the changes in the ubiquitin chain topology caused by ubiquitination could change protein stability, membrane transport, and cellular localization. USP9X in the DUBs (ubiquitin-specific protease family) could bind to and deubiquitinate AMOT. Finally, inhibit the expression activity of YAP/TAZ [28]. Transmembrane proteins (Crumbs, $\mathrm{Crb})$ participate in regulating the polarity of the top-bottom layer of the cell. They could bind to Expande (Ex) through the FBM region to change its subcellular location and stability, thereby regulating the expression of swimming genes [29]. Merlin (Mer) could form a complex with Ex and enriched in the top layer of epithelial cells. It could cooperate with Kibra to bind SAV1 and participate in the signal transduction of the Hippo pathway [7,30]. Also, the aPKC complex formed by aPKC, Bazooka, and Par6 could antagonize Scrib to promote the activation and expression of YAP/Hippo [31].

\section{Regulation of Planar Cell Polarity on the Hippo signaling pathway}

The three-dimensional morphological

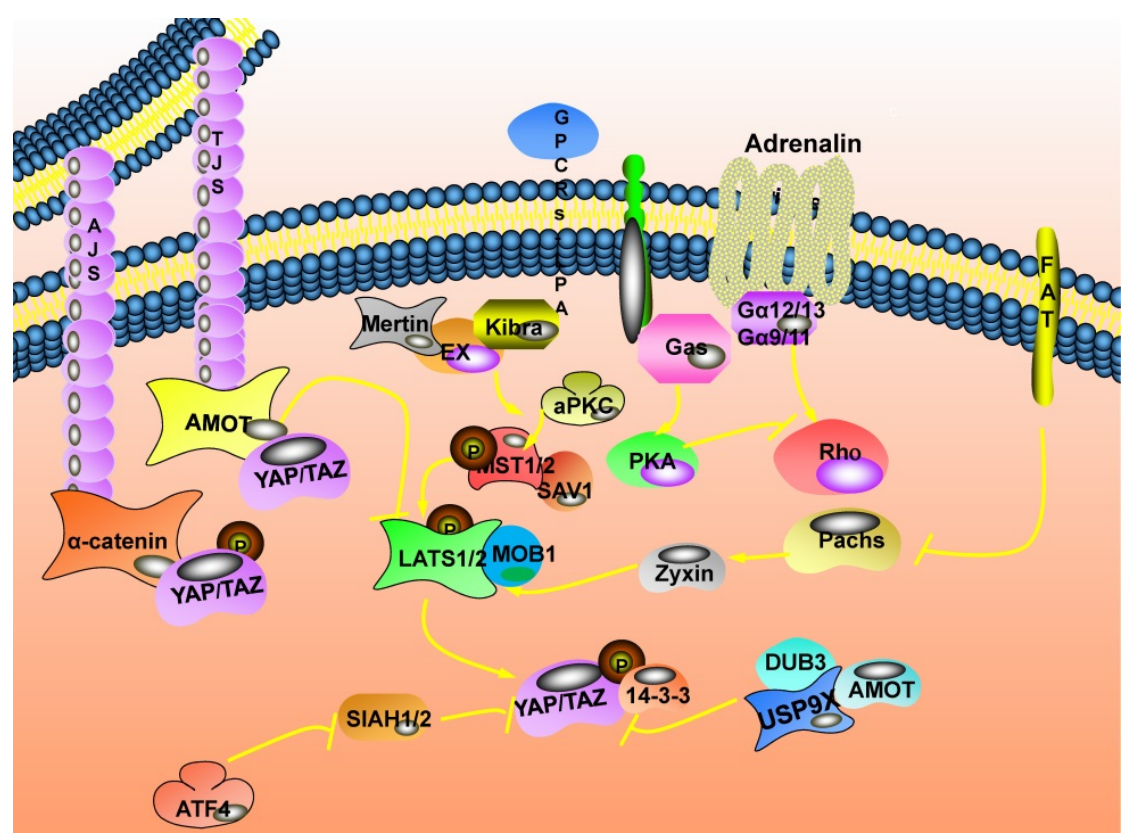

Figure 2. The upstream regulatory network of the Hippo signaling pathway. Cell junction related proteins are located on the cell membrane. Mainly divided into tight junctions (TJs) and adhesive connections (AJs). Ubiquitin specific peptidase 9 X-linked (USP9X) in the ubiquitin-specific protease family (DUBs) could bind to and deubiquitinate angiomotin (AMOT). FAT atypical cadherin 1 (FAT) is the first transmembrane receptor identified to affect the expression of the Hippo pathway, and it is also a transmembrane apex determinant. The mechanism by which FAT affects the expression of the Hippo pathway depends on the relationship between FAT and Expande (Ex). distribution of cells in tissues usually shows axiality, and the distribution is not random or uniform. When cells' subcellular structure is distributed asymmetrically along one or several axes, this phenomenon is called cell Polarity [32] Planar Cell Polarity (PCP) also affects the expression of the Hippo pathway [33,34]. Atypical Cadherin plays an essential role in the process of biological development and tissue morphogenesis. FAT is the first transmembrane receptor identified to affect the expression of the Hippo pathway, and it is also a transmembrane apex determinant [35].

Here, the mechanism by which FAT affects the expression of the Hippo pathway depends on the relationship between FAT and Ex: When the FAT, Ex and Hippo pathways are regulated in a linear cascade, Fat mediates the Hippo pathway by affecting the subcellular localization and stability expression of Ex [36]; when FAT and Ex are in parallel and coordinated regulation, it directly promotes the phosphorylation of LATS1/2 to mediate the expression of the Hippo pathway [37]. Besides, FAT could also change the stability of LATS1/2 through Dachs and Zyxin to mediate the expression of the Hippo pathway [38].

\section{Regulation of cell membrane receptors on the Hippo signaling pathway}

The receptors distributed on the cell membrane will activate or inhibit the relevant signal pathways when they feel external stimulus signals and affect cell proliferation or apoptosis [39]. G protein-coupled receptors and insulin receptors are essential cell membrane receptors that mediate extracellular signal transmission into the cell [40]. The Hippo pathway is regulated by upstream membrane protein receptors and could respond to extracellular growth signals [41]. The serum proteins in the serum could bind to their G ProteinCoupled Receptors (GPCRs) on the membrane and activate the expression of YAP/TAZ through Rho GTPase [42]. Thrombin could promote the conversion of soluble fibrinogen into insoluble fibrin. It could also activate their $G$ proteincoupled receptor-protease activated receptor (PARs) on the cell membrane and activate the expression of YAP/TAZ [43]. The crosstalk mechanism between insulin and insulin like growth factor 1 (IGF-1) signaling pathway in 
pancreatic cancer inhibits the expression of phosphatidylinositol 3-kinase (PI3K) explicitly, effectively reducing YAP/TAZ [44]. In addition, vascular endothelial growth factor (VEGF) could activate VEGF receptors and inhibit the phosphorylation of LATS1/2 and YAP [35].

\section{Regulation of the cellular mechanical environment on the Hippo signaling pathway}

The cellular mechanical environment includes various physical forces that may impact the cell and other surrounding environments. This may affect the internal or external forces generated by the cell. Depending on the composition of the tissue and surrounding matrix, the hardness of the matrix, the size of the available space, as well as the considerable changes in the cell microenvironment throughout the body, the cellular mechanical environment play a significant part in the process of cell proliferation, survival and differentiation, tissue regeneration and wound repair [22]. Abnormal mechanical transduction may lead to the activation of key signaling pathways and trigger abnormal cell behavior, potentially affecting the development of chronic diseases [45]. The downstream effector YAP/TAZ in the Hippo signaling pathway not only responds to mechanical stimuli (such as tissue stretching, cell density and area, cell adhesion and extension, cell contact, and extracellular matrix hardness, etc.) but also an essential regulator of cell response to these stimuli [46].YAP/TAZ translates a series of signals from splicing pressure to cell morphology and extracellular matrix hardness and converts them into cell-specific transcriptional programs. This mechanism of transduction is essential to drive stem cell behavior and regeneration [47].

It is worth noting that YAP/TAZ's various mechanical signal regulators are mostly concentrated in the actin cytoskeleton. Actomyosin is the main component of cells that respond to mechanical stimuli. The joint action of the nuclear skeleton linker and cytokine mediates mechanical force transfer to the nucleus through actin cells [48]. When cells grow at high density, the connections between cells increase, and the upstream kinase LATS1/2 of the Hippo signaling pathway is activated, causing YAP/ TAZ phosphorylation and failing to enter the nucleus. In addition, when the cells growing on the soft substrate do not have enough area to stretch, the mesenchymal stem cells will differentiate into adipocytes. The cell morphology becomes round, and YAP/TAZ is mainly distributed in the cytoplasm; on the contrary, mesenchymal stem cells differentiate into osteoblasts, and the cell morphology is flat, YAP/TAZ mainly located in the nucleus $[47,49]$.
However, the interaction between the Hippo pathway and cell tension still needs further exploration. Sun et al. proposed a YAP/TAZ mechanical sensing calculation model, exploring the multi-scale relationship between the adhesion signal and the expression abundance of YAP/TAZ. This model converts the mechanical properties of the extracellular matrix into biochemical signals through adhesion and cascades intracellular signals related to cytoskeleton dynamics to perform molecular level disturbance and sensitivity analysis. It provides an analytical platform for looking at YAP/TAZ activity carefully in the context of combining varying signal pathways [50].

\section{Regulation of oxidative stress on the Hippo signaling pathway}

Oxidative stress is the state of imbalance between oxidation and antioxidant when cells need antioxidants to neutralize excessive free radicals [51]. Protein kinase AMP-activated catalytic subunit alpha 1 (AMPK) is a crucial regulator of bioenergy metabolism, and the increase of adenine phosphoribosyl transferase (AMP)/ATP content in the body could activate AMPK [52]. Under hypoxic conditions, the content of ATP decreases, and the cells are in an energy-deficient state. There are two molecular mechanisms for activated AMPK to regulate the activity of YAP. One is that AMPK activates and directly phosphorylates the S61 and S94 sites of YAP. Phosphorylation at S94 inhibits the binding of YAP to TEAD, and the expression of downstream target genes of YAP is inhibited [53]; the other is that AMPK promotes the expression of LATS2 through phosphorylation of angiomotin like 1 (AMOTL1) and activates the Hippo signaling pathway to cause YAP phosphorylation and inactivation [54].

Hypoxia is a stress condition closely related to pathological processes such as tumor development and myocardial injury. Under hypoxic conditions, the E3 ubiquitin ligase SIAH2 could bind to LATS2 and undergo ubiquitination modification. The expression of LATS2 is degraded, thereby activating YAP/TAZ into the nucleus and increasing the secretion of PGE2 (prostaglandin 2) [55]. YAP is down-regulated in neuroma blasts after brain hypoxia-reoxygenation (HR) injury. Overexpression of YAP could block the apoptotic signal associated with mitochondria and inhibit HR-mediated neuroma cell death [56]. The upstream kinase MST1 plays an important role in reactive oxygen, which could induce cell death, reactive oxygen defense, and could be activated under oxidative stress conditions $[2,25,26]$. Under prooxidant conditions, activating transcription factor 4 
(ATF4) inhibits the expression of E3 ubiquitin-protein ligase Nedd-4 (NEDD4.2) and WW domain containing E3 ubiquitin protein ligase 1 (WWP1) in mRNA levels, and the LATS1 proteasome-mediated by encoding ubiquitin ligase would be degraded [57]. As a transcriptional coactivator of forkhead box $\mathrm{O} 1$ (FOXO1), YAP could directly bind to FOXO1 and activate FOXO1-mediated transcription of catalase and superoxide dismutase 2 (SOD), reducing heart damage by oxidative stress and ischemia-reperfusion [58].

\section{Regulation of Hippo signaling pathway by virus}

A virus is a non-cellular life form with a simple structure containing only one nucleic acid (DNA or RNA), which replicates, transcribes, and translates in the host cell. When it enters the host cell, self-replication begins and affects the gene expression and energy metabolism of the host cell. Hippo signaling pathway also plays a critical negative regulatory role in virus-induced diseases. Many human viruses exert their carcinogenic functions by activating the activities of YAP and TAZ. One study found that Human papilloma virus (HPV) virus induces degradation of Scribble protein by HPV E6 oncoprotein, which leads to the development of

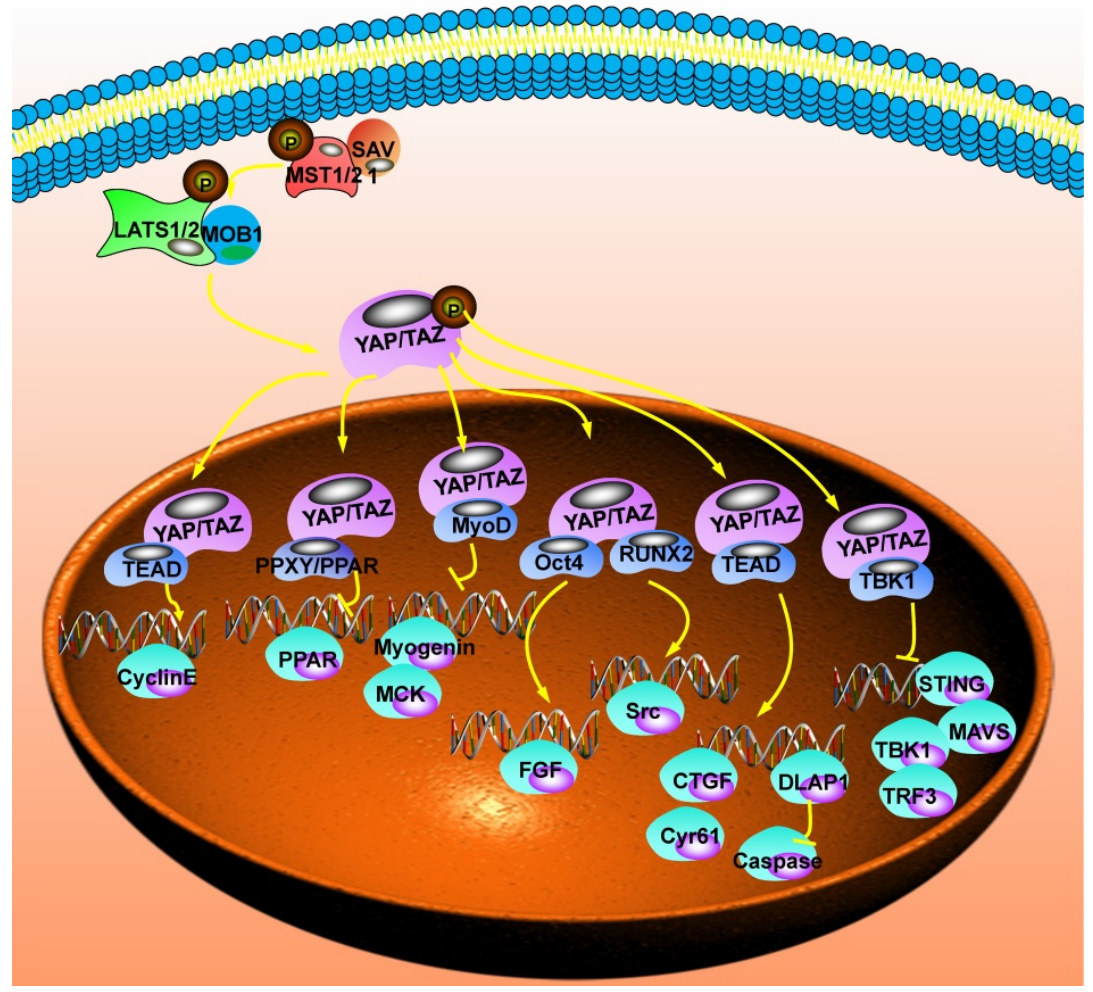

Figure 3. The downstream regulatory network of the Hippo signaling pathway. After YAP enters the nucleus, it needs to be combined with other proteins to form a complex before it could regulate transcription. After that, it could inhibit the transcription of peroxisome proliferator activated receptor (PPAR), creatine kinase (MCK), non-receptor tyrosine kinase (Src) and stimulator of interferon response cGAMP interactor 1 (STING). It could also promote the transcription of Cyclin E, epidermal growth factor (EGF), Connective tissue growth factor (CTGF), cellular communication network factor 1 (CCNI) and Drosophila melanogaster (DLAPI). cervical cancer [59]. In addition, YAP is more likely to localize to the nucleus in HPV-positive oropharyngeal squamous cell carcinoma than in precancerous tissues, which is also associated with the development of squamous cell carcinoma of the oropharynx [60]. HBV recognized by Toll-like receptors can activate NF-kB and Hippo signaling in both murine and human hepatocytes, thereby inducing innate immune mechanisms [61]. In addition, EBV-infected gastric cancer cells can be observed to have greatly increased YAP activity and elevated expression of downstream target genes of the Hippo signaling pathway after 12-24 hours. Meanwhile, in breast cancer, EBV infection also causes inactivation of the Hippo signaling pathway [62]. It also has been reported that when hepatitis $\mathrm{C}$ virus (HCV), infectious mollusc virus (MCV), mouse polyomavirus (MupyV), Kaposi Sarcoma-associated herpesvirus (KSHV), Zika virus (ZIKA) affecting host cells, the increase of YAP/TAZ expression activity could be found [63].

\section{The downstream regulatory network of the Hippo signaling pathway}

YAP/TAZ, the Hippo signaling pathway's main effector, does not contain DNA binding sites and is a cofactor for transcription activation. Combining with TEADs, p73, erb-b2 receptor tyrosine kinase 4 (ERBB4), early growth response 1 (EGR-1), T-box transcription factor 5 (TBX5), SMADs, and other transcription factors could activate the transcription of downstream target genes. Therefore, its transcriptional association domain determines the biological function of YAP/TAZ and the selection of its target genes. The functions of interacting proteins are different, and the downstream mechanisms of YAP/TAZ involved in regulation are also different [64] (Fig. 3).

\section{Regulation of Hippo signaling pathway on cell growth}

Connective tissue growth factor (CTGF) is the direct target gene of YAP-TEAD. There are 3 TEAD binding sites in its promoter sequence, making TEAD directly bind to the CTGF promoter [65]. In breast cancer cells, the anti-apoptotic proteins $\mathrm{Bcl}-\mathrm{xL}$, cIAPI, and survivin are activated by CCN1 and CTGF, which could antagonize taxane-induced apoptosis. Similarly, the promoter activity of 
YAP/TAZ is activated by CCN1 and CTGF, which could antagonize the function of taxane [66]. The PDZ binding motif plays an important role in the biological functions of transcription coactivators. The loss of the PDZ binding motif could considerably prevent the oncogenic transformation induced by YAP (5SA) and the nuclear localization of CTGF expression. This effect is mediated by TEAD-mediated CTGF transcription [67].

Tissue fibrosis is a pathological status that is connected to the impaired epithelial repair and excessive deposition of extracellular matrix (ECM). In fibroblasts, ECM stiffness could mechanically activate the expression of YAP/TAZ, thereby promoting the production of fibrotic mediators and fibroblast growth factor (FGF), leading to tissue stiffness, and establishing a feedforward loop for fibroblast activation and tissue fibrosis [68]. VEGF plays a crucial function in organ development and early tumor angiogenesis, promoting its growth and spread. YAP/TAZ mediates the signal transduction of VEGF. Simultaneously, the VEGF signal affects GTPase activity, which contributes to the activation of YAP/TAZ. This positive feedback control leads the changes in transcription and cytoskeleton, and finally promotes tumor cell angiogenesis [68].

\section{Regulation of Hippo signaling pathway on cell differentiation}

The differentiation of myoblasts is essential for forming skeletal muscle and is regulated by a series of transcription factors (including myogenic differentiation factor 1 (MyoD), Myogenin and transcription co-regulators). Through the interaction between the WW and MyoD, TAZ (transcriptional coactivator with PDZ binding motif) could strengthen the binding of MyoD and myogenin gene promoter, which could activate and promote the transcription of myogenin and MCK genes. Finally, the terminal differentiation of muscle cells is induced. The MEK5/ERK5-MAPK cascade is essential for muscle cell differentiation. Muscle differentiation could promote by YAP by activating the Abl/Src/MEKK3/ MEK5/ERK5 kinase cascade [69]. The high activity of YAP in muscle fibers will not cause fiber hypertrophy or fiber type changes but will cause reversible atrophy and degeneration [70]. As a necessary transcription factor, RUNX2 could stimulate bone formation and promote bone cell differentiation in combination with TAZ. The YAP molecular structure has an SH3 binding domain, which TAZ does not have. It could make YAP1 directly bind to Src. When the activity of Src is inhibited, it could induce osteoblast differentiation.

There are currently two research results. One is that YAP1 acts as a transcriptional co-repressor of RUNX2 on the osteocalcin promoter in response to Src stimulation, which mediates the phosphorylation of YAP tyrosine sites. And this could promote the binding of YAP and RUNX2. The other is that YAP located in the nucleus, acts as a transcriptional coactivator of RUNX2, and promotes bone formation [71]. PPARY is a ligand-activated receptor in the nuclear hormone receptor family. PPARY is a ligand-activated receptor in the nuclear hormone receptor family. As an important cell differentiation transcription factor, PPARY has two molecular mechanisms involved in regulating the Hippo signaling pathway. One is that TAZ directly binds to the PPXY motif of PPARY to inhibit the active expression of PPARY. The other is the upstream kinase SAV1 competing with TAZ to antagonize the same PPXY motif that binds to PARY. At the same time, SAV1 promotes the phosphorylation of TAZ through the Hippo pathway. The retention of TAZ in the cytoplasm inhibits the nuclear binding of TAZ and PPARY [72]. Notch signal is a critical factor in epidermal differentiation, triggering proliferation, or cell differentiation in different cell types. Cell morphology and extracellular matrix could regulate the activity of YAP/TAZ, and mechanical activation of YAP/TAZ could inhibit the differentiation of epidermal stem cells by inhibiting Notch signaling [73].

\section{Regulation of Hippo signaling pathway on apoptosis}

The Hippo signaling pathway regulates the drosophila apoptosis inhibitor (DIAP1), which mediates caspase's inactivation to inhibit cell apoptosis [58]. At the same time, microRNA Bantam in Drosophila is also a downstream target gene of the Hippo signaling pathway, regulating growth and tumorigenesis by inhibiting the translation of the apoptotic factor- Hid [74]. Also, YAP1 regulates anaplastic lymphoma kinase (ALK) by inhibiting the anti-apoptotic factors MCL1 apoptosis regulator and Bcl-xL (B cell leukemia/lymphoma), which has clinical significance for molecular targeted therapy in non-small cell lung cancer with ALK-positive mutations. In terms of the cycle, the Hippo pathway is highly inactivated in glomerular mesangial cells cultured in high glucose in vitro. The reduction of MST1/2 and LATS1/2 phosphorylation promotes YAP expression and nuclear entry. It increases the YAP-TEAD interaction and activates the expression of downstream target genes such as cyclin E [75]. In embryonic neuroblasts, the Ama-Nrt-Abl pathway could also regulate cyclin E expression by changing the function of the Hippo pathway effector YAP [75]. 


\section{Regulation of Hippo signaling pathway on cellular immune factors}

Hippo signaling pathway plays an integral part in the immune system and a variety of functional immune cells, especially in the process of immune cells responding to the virus, bacterial invasion, or tumorigenesis and maintaining their homeostasis. In terms of antiviral defense, YAP/TAZ could form a complex with TANK-bindingkinase1 (TBK1) and effectively prevent the K63 ubiquitination modification of TBK1. Moreover, it also could form STING/MAVS-TBK1-IRF3 complex [76]. In terms of immunity, YAP/TAZ often does not require the regulation of upstream kinases and directly affects $T$ cells. At the same time, it could regulate the crosstalk between immune cells and tumor cells in the tumor microenvironment by inhibiting the polarization of bone marrow cells and macrophages. Besides, the important immunosuppressive factor PD-1 could bind to the upstream kinase MOB1 of the Hippo pathway and inhibit MOB1 phosphorylation. The downstream targets of the Hippo pathway, CYR61, and CTGF, are directly involved in immune regulation mediated by PD-1 [76].

\section{Tumor phenotypes affected by the Hippo signaling pathway}

When external signals activate the Hippo signaling pathway, it may inhibit cell proliferation and promote cell apoptosis, which could also control the size of tissues and organs and inhibit tumor formation. When the Hippo signaling pathway is abnormal, it will participate in the signal transduction of Wnt, Notch, EGFR, TGF- $\beta$, JAK-STAT, and other pathways. Thereby, it could affect the occurrence and the development of various human tumors, such as lung cancer, breast cancer, gastric cancer, hepatocellular carcinoma, renal cell carcinoma, colorectal cancer, etc.

\section{Hippo signaling pathway promotes cell proliferation}

Cell proliferation is an essential feature of organisms. Cells divide to produce new cells to supplement aging or dead cells in the body. The strict control of cell division is essential to maintain the normal development of cells and the homeostasis of the cell environment. Abnormal cell proliferation is related to a variety of pathological conditions. The Hippo signaling pathway plays a significant part in regulating cell proliferation without relying on organ size control. Studies on Drosophila/mouse have found that the inactivation of tumor suppressor genes in the Hippo signaling pathway or the activation of the oncogene Yki/YAP will lead to excessive tissue growth. The characteristic performance is increased cell differentiation and inhibition of cell apoptosis.

The immunohistochemical method could be used to detect the expression of YAP in non-small cell lung cancer cases. The results showed that YAP was highly expressed in the nucleus in adenocarcinoma, and it was positively correlated with the expression of cyclin A and mitogen-activated protein kinase. The pathological TNM staging study of squamous cell carcinoma found that the expression of YAP in the cytoplasm of stage I was higher than that of stages II to IV. It suggests that the high expression of YAP in the nucleus is closely related to the cell cycle, promoting the proliferation of renal cancer cells. This indicated that the high expression of YAP in the cytoplasm could be used as an independent predictor of clinical pathological staging. After overexpression of MST1 in lung adenocarcinoma A549 cell line, cell growth was inhibited, and the anti-proliferation ability was related to YAP phosphorylation. After the YAP gene was explicitly knocked out, the proliferation of hepatocytes was inhibited, and even necrosis occurred. Unusual concentrations of bile acids could perform the role of upstream activators of the Hippo signaling pathway, promote the expression of scaffold protein IQGAP1, and activate YAP transcriptional activity to stimulate liver growth and tumorigenesis [77].

\section{Hippo signaling pathway inhibits cell apoptosis}

Apoptosis, also known as programmed cell death (PCD), refers to the autonomous and orderly death of cells under pathological and physiological conditions, in order to maintain a stable biological environment [78]. In hepatocellular carcinoma and intrahepatic cholangiocarcinoma cells, YAP and survivin are highly expressed in nuclei. Moreover, survivin is an inhibitor of apoptosis protein, and the expression of survivinm RNA depends on YAP protein [79]. After overexpression of MST1 in vitro, YAP phosphorylation increased, and mRNA expression of ETGF, amphiregulin (AREG), and survivin was all down-regulated. After that, cell proliferation was inhibited, and apoptosis was induced [80]. After knocking down the TAZ gene in vitro, the expression of cyclin A and CTGF decreased significantly, the cell cycle was arrested in the G0 G1 phase, and cell proliferation was inhibited. At the same time, it was accompanied through a considerable rise in the expression of caspase 3 and induced apoptosis, showing the ability of TAZ to promote cell proliferation and inhibit cell apoptosis $[81,82]$. 


\section{Hippo signaling pathway promotes cell invasion and metastasis}

Cell migration is the basis for many important physiological activities of cells and an important step and key link in pathological processes such as inflammation and tumorigenesis [83,84]. Enhanced YAP/TAZ activity could promote tumor cell migration [85]. As a phospholipid messenger between cells, lysophosphatidic acid (LPA) could activate G protein-coupled receptors and take part in the normal physiological functions of the body. In ovarian cancer, the Hippo signaling pathway is complicated in LPA-mediated migration of ovarian cancer cells, manifested by LPA-induced dephosphorylation of YAP into the nucleus to function. This response relies on the participation of multiple regulatory factors such as Rho/Rock, PP1A, AREG, and EGF. Using siRNA knockdown YAP, YAP expression activity decreases, and LPA-induced ovarian cancer cell metastasis and invasion ability are also significantly reduced [82].

TEAD is the downstream effector YAP/TAZ of the Hippo signaling pathway. The interacting protein is essential for YAP/TAZ-mediated tumor growth and metastasis. The invasion ability of metastatic breast cancer is closely related to TEAD transcription

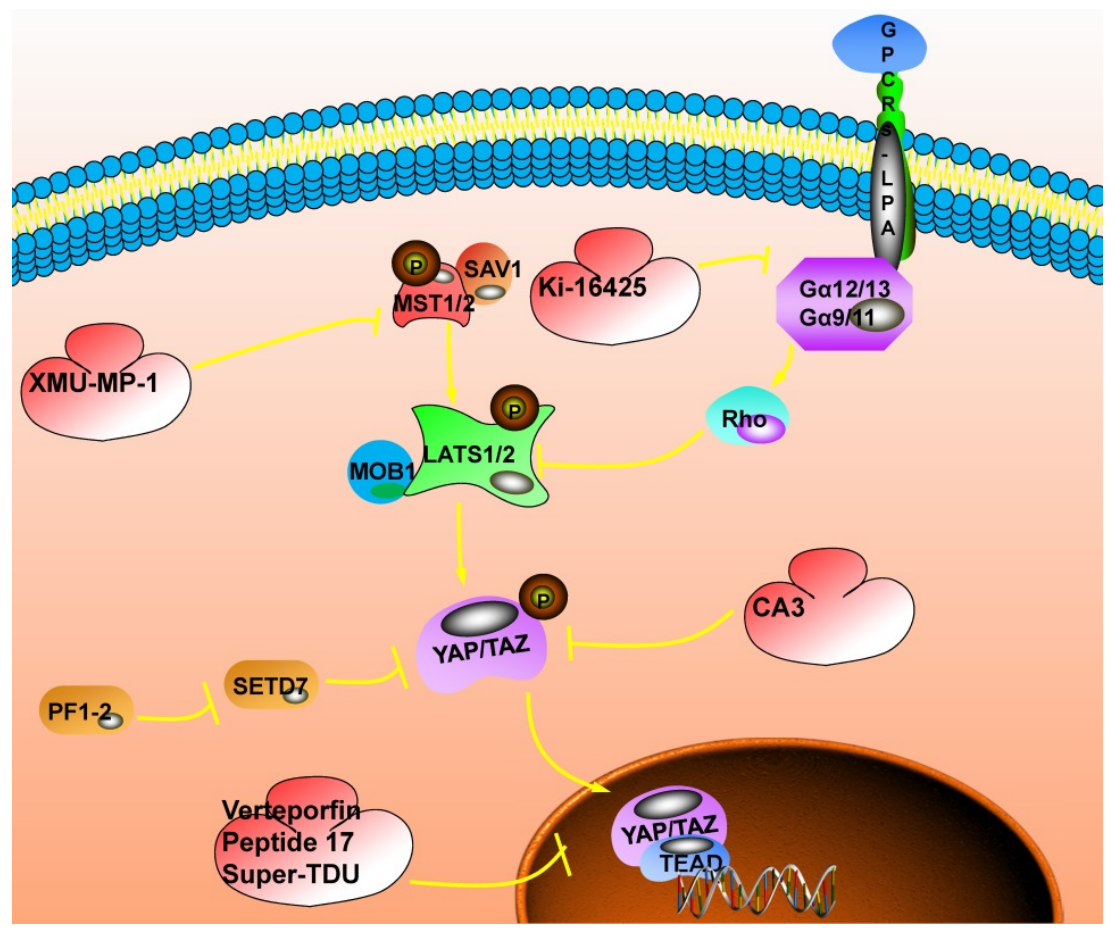

Figure 4. The inhibitory target involved in the Hippo signaling pathway. The switch that closes the Hippo pathway is whether YAP enters the nucleus. When YAP is phosphorylated, it will be stagnated in the nucleus and the Hippo pathway will be activated; when YAP enters the nucleus, the Hippo pathway will be closed, which could promote the occurrence of tumors. The current targeted inhibitors have two ideas, one is to promote the phosphorylation of YAP. For example: XMU-MP-1 could inhibit the phosphorylation of MST1/2, promote the cascade of MST1/2, LAST1/2, and YAP, and promote the phosphorylation of YAP; Ki-16425 could inhibit the phosphorylation of LAST1/2 and promote the phosphorylation of YAP; CAS could directly promote phosphorylation of YAP. Another idea is to inhibit the interaction of YAP with TEAD after entering the nucleus. Here, Verteporfin compounds could be used to achieve this function. activity. In non-metastatic tumor cells, enhancing the transcriptional activity of YAP-TEAD could lead to non-metastatic NMuMG cells, 67NR cells, and A375 cells to metastasize to the primary site [86]. In breast cancer cells, overexpression of TAZ can regulate cell proliferation, migration, and epithelial-mesenchymal transition. The interaction of TAZ-TEAD enhances the activity of the bone morphogen-netic protein 4 (BMP4) promoter, and the activated BMP4 also, in turn, increases the expression activity of TAZ. Western blot and wound healing analysis showed that TAZ overexpression could induce cell migration and increase. pSmad1/5 expression activity while knocking out the target gene BMP4 gene significantly reduces cell migration ability $[87,88]$.

\section{Possible tumor suppressor targets in the Hippo signaling pathway}

The subcellular localization of YAP/TAZ is a key determinant of its transcriptional regulation and signal transduction, which provides an attractive target for cancer therapy. YAP/TAZ is often over-activated due to the disorder of the Hippo signaling pathway. It has been confirmed that the activation or overexpression of YAP/TAZ is related to the poor prognosis of cancer patients. The inhibition of YAP/TAZ could improve the prognosis of cancer patients. Therefore, the development of YAP/ TAZ inhibitors based on the Hippo signaling pathway is expected to become a cancer treatment target (Fig. $4)$.

\section{Targeting inhibition of upstream kinase molecules in the Hippo signaling pathway}

It is difficult to use small molecule target the unstructured nature of YAP and TAZ. Therefore, YAP/TAZ inhibitors tend to target the upstream or downstream molecules of YAP. Here we divide inhibitors into two categories, one is upstream-targeting inhibitors, and the other is downstream-targeting inhibitors. Here we focus on targeting upstream inhibitors, and the next section on targeting downstream.

MST1/2 is an essential cascade kinase in the Hippo signaling pathway, and XMU-MP-1 is a highly effective and selective inhibitor of MST1/2 (MST1, IC50=71.1nM; MST2, IC $50=38.1 \mathrm{nM})$. In vitro studies have 
shown that in mouse models of acute or chronic liver injury, the addition of XMU-MP-1 can effectively activate the protein expression of the downstream effector YAP, thereby enhancing intestinal repair in mice and promoting liver repair and regeneration[89]. In the study of head and neck squamous carcinoma, it was found that FAT1 is directly related to MST1, the assembly of the Hippo kinase core complex, which leads to the subsequent phosphorylation of LATS1/2 and YAP [89].At the same time, PIK3CA could activate YAP/TAZ through the PIK3-PDK1 axis to mediate different stimuli (EGFR, FAK, fibronectin, GPCRs, etc.) and inhibit some BET protein inhibitors such as Birabresib, which inhibit the bromine domain and extra terminal domain of the protein. This is expected to become a new target for blocking the Hippo signaling pathway to treat cancer.

In addition, some molecules that target surface receptors could also inhibit the activation of the Hippo signaling pathway. Such as EGFR-targeting inhibitors, erlotinib [90,91] and AG-1478 [92]; G-protein-targeting coupled receptors, losartan and dihydrohexidine; targeting of antibody-BHA 2.1 [93] and clone AIIB2 [94], RGD peptide [95] and cyclic RGD peptide [96]; Inhibitors targeting VEGFR, such as SU4312, apatinib, axitinib and pazopanib, and the forskolin. Some actin-targeted inhibitors that can promote YAP nuclear localization can also be used to block YAP from entering the nucleus, such as latrunculin A [97], cytochalasin D [98,99], blebbistatin and ML-7. In the same way, okadaic acid or calmodulin A can over-express the phosphatase that helps phosphorylate YAP, thereby trapping more YAP in the cytoplasm.

Some metabolism-related molecules have also been used as targets to block the Hippo signaling pathway. The activity of YAP can be inhibited by targeting focal adhesion kinase (FAK), Src and integrin-linked kinase (ILK). In addition, AMPK activator A769662 and AICAR (an AMP mimic) are also inhibitors of YAP [100-102].

\section{Targeting the inhibition of downstream transcription factors in the Hippo signaling pathway}

The downstream effector YAP of the Hippo pathway requires the coordination of transcription factors to regulate cell proliferation, anchorageindependent growth, and epithelial-mesenchymal transition. Among them, TEAD is an important binding partner of YAP to exert its carcinogenic activity. Inhibition of YAP/TEAD activity in vivo could effectively inhibit the biological functions caused by the YAP-TEAD interaction. The introduction of cysteine at positions 87 and 96 of YAP could induce disulfide formation, and the change of the peptide significantly improves the ability to disrupt the YAP-TEAD interaction in vitro. Similarly, introducing a dominant-negative mutation $(\mathrm{Y} 406 \mathrm{H})$ of TEAD could also disrupt the YAP-TEAD interaction. At this time, the tumor growth rate in the hepatocellular carcinoma xenograft model was significantly reduced, confirming that it is an effective method to prevent the formation of the YAP-TEAD complex by directly targeting TEAD [103].

In addition, in vitro selection of appropriate inhibitors for YAP-TEAD interaction is also a useful breakthrough point [104,105]. The small molecule compound Verteporfin (VP) could inhibit the YAPTEAD interaction and show a significant inhibitory effect on TEAD transcription activity. At the same time, the expression level of YAP/TAZ is also significantly reduced [106]. VP arrests the cell cycle in the G1 phase and induces apoptosis in a dose- and time-dependent manner. Mechanism studies have shown that VP damages the YAP-TEAD interaction, thereby inhibiting the expression of target genes and inhibiting cell proliferation [107]. Peptide17 also showed a strong affinity with TEAD in vitro experiments, effectively destroying the interaction of YAP-TEAD, and has its potential application value in YAP-related cancers [108]. An FDA-approved verteporfin drug could also inhibit the YAP-TEAD interaction and inhibit YAP-induced overgrowth of cancer cells in vivo and in vitro.

The TDU domain of vestigial like family member 4 (VGLL4) is a natural antagonist of YAP, which competes with YAP to bind TEAD [109]. Super-TDU could specifically target YAP/VGLL4 tumor cells with a high ratio, reduce the endogenous YAP-TEAD interaction, and down-regulate the expression of target genes such as CTGF, CCN1, and caudal type homeobox 2 (CDX2) [110,111]. Carbonic anhydrase 3 (CA3) prevents the transcriptional activity of YAP-TEAD explicitly. In esophageal adenocarcinoma cells, the combination of $\mathrm{CA} 3$ and 5-FU could synergistically inhibit YAP expression and the growth of esophageal adenocarcinoma cells. It indicates that CA3 is a new inhibitor of YAP $[111,112]$. In addition, drugs targeting downstream YAP/TAZ targets (BCL-xL, FOXM1 and TG2) could also be used to combat YAP/TAZ-mediated carcinogenesis, such as A37 [113], celecoxib [114], TP-0903 [115], cyclic peptide RA-V (deoxybouvardin) [116], navitoclax [117], thiostrepton [118] and NC-9 [119,120].

\section{Molecular inhibition of targeting Hippo signaling pathway}

The occurrence of cancer will show changes in various signaling pathways, and these signaling 
pathways have modes of co-occurrence or mutual exclusivity. $89 \%$ of tumors have at least one driving change in these pathways, which reminds new ideas for combination therapy in cancer. The methyltransferase SET domain containing 7 (SETD7) plays a central regulatory function in the Hippo/YAP pathway and $\mathrm{Wnt} / \beta$-catenin pathway to control tumors' occurrence. Similarly, YAP is also involved in the activation of the Wnt pathway. PFI-2 is a highly effective and selective inhibitor of SETD7 methyltransferase. In (R)-PFI-2 pretreated human breast cancer cell MCF7, YAP's location changed rapidly. It reveals the effect of the lack of phenotypic SETD7 on the Hippo signaling pathway, indicating that the methyltransferase activity of SETD7 continuously and dynamically regulates YAP [112].

Lysophosphatidic acid (LPA) is a biologically active lysophospholipid and participates in a variety of physiological reactions. In studying LPA receptors' functional expression in salivary gland epithelial cells, it was suggested that LPA receptors could activate the expression of downstream effectors YAP/TAZ through LATS/MOB1 and RhoA/ROCK. Up-regulation of the expression activity of the downstream genes CTGF, NKRD1, and CCN1 targeted by YAP/TAZ were observed in LPA-treated cells [121]. In the study of LPA-induced cell contraction involved in the extracellular matrix (ECM), it was found that LPA and its receptor promote the YAP/TAZ transcriptional activity in vitro by regulating cell contraction tension and enhance the expression of CTGF, which in turn leads to ECM increase [122]. Ki-16425 is a competitive and effective LPA reversible inhibitor. In the cell line, it could block LPA receptors to induce the dephosphorylation of YAP/TAZ and inhibit the Hippo pathway. However, the Hippo signaling pathway is critical to physiological homeostasis and regeneration after tissue injury. Tumor treatment drugs for Hippo pathway inhibition may cause potential damage to patients with co-morbidities. Therefore, caution should be taken in the selection and dosage of Hippo pathway inhibitors [123,124]. Unlike bulk materials, nanoparticles exhibit unique properties such as large specific surface area, electromagnetic properties, and optical behavior. These characteristics enable them to solve many problems faced by traditional diagnosis and treatment methods, which are mainly manifested in the following aspects: First, during the preparation process of nanoparticles, the composition, size, shape, and surface properties could be adjusted artificially, so that nanostructures with specific properties could be synthesized as needed to encapsulate and protect anti-cancer drugs. Secondly, by controlling the shape, size, and surface modification of nanoparticles, the drug-loaded nanoparticles could be targeted to reach specific lesions. They could release drugs in a controlled manner without affecting normal tissues and organs. This solves the problem of traditional therapies, including the systemic toxicity and low treatment efficiency. Finally, some nanoparticles have excellent magnetic or optical properties. They could be used for biological imaging (such as magnetic resonance imaging or fluorescence imaging, etc.) to integrate diagnosis and treatment in the body. However, as of now, no nano-drug targeting the Hippo pathway has been developed. It could be hoped that future Hippo pathway research could also focus on nano-strategies. However, so far, no nano-drug targeting the Hippo pathway has been developed, and it is hoped that future Hippo pathway research could focus on nano-strategies.

\section{The other biological effects of the Hippo signaling pathway}

In addition to tumor-related regulation, the Hippo pathway is also closely related to many other biological processes. In recent years, in the Hippo signaling pathway's research directions, the focus of research has shifted from the initial screening of core regulatory factors to functional biological research, which is mainly reflected in organ development, tissue regeneration, stem cell self-renewal, tumor occurrence and development.

\section{The Hippo signaling pathway involved in regulating organ size}

Hippo signaling pathway participates in and mediates the development of human organs and carcinogenesis [112,125]. For example, after specific overexpression of YAP in transgenic mice's liver, abnormal liver enlargement was observed. After the overexpression of YAP was terminated, the liver's specific gravity slowly returned to a normal level. Similarly, by specifically knocking out the upstream kinase MST1/2, SAV1, or neurofibromin 2 (NF2) in the Hippo pathway, abnormal liver enlargement was observed. However, changes in the Hippo signaling pathway will not cause changes in all organs. For example, after specifically knocking out MST1/2 in mice, except for abnormal hyperplasia of liver, heart, stomach, and spleen, there is no apparent hyperplasia of kidney, lung, and limbs. While specifically knocking out YAP expression in the intestine, no obvious defects were found in the intestinal tissue structure and size. Besides, the loss of YAP function has no effect on breast development during adolescence, but it will seriously hinder the growth and development of the breast during pregnancy. These studies suggest that the Hippo signaling 
pathway is involved in regulating organ size and depends on the type of organ.

\section{The Hippo signaling pathway involved in regulating tissue regeneration}

Under physiological and pathological conditions, the key to wound healing is the generation and maintenance of blood vessels. YAP/TAZ participates in the formation of endothelial cells, vascular barriers, and vascular remodeling. The regulation of angiogenesis plays a central role in the restoration of the body after the treatment of various diseases. Therefore, although the normal growth and development of tissues and the maintenance of homeostasis do not depend on the active expression of $\mathrm{YAP} / \mathrm{TAZ}$, tissue regeneration after tissue injury needs to depend on the active expression of YAP/TAZ [126,127]. For example, nude mice injected with dextran sodium sulfate-induced colitis could effectively regenerate and repair after a period of feeding. While YAP expression in nude mice is specifically knocked out, it cannot effectively regenerate and repair after a period of feeding. Similarly, after partial excision of $70 \%$ of the mouse liver, the liver with strong regenerative capacity can also use the remaining part to regenerate and recover. During this period, YAP's expression level and activity increased significantly and remained at a high level for a long time. MST1/2 and LATS1/2 expression activity decreased. When the liver regeneration is restored, the activities of YAP, MST1/2, and LATS1/2 return to the level of the resting state of the liver, which indicates that the Hippo signaling pathway is significant for maintaining a typical liver weight ratio and liver homeostasis. Compared with intestinal tissue and liver, adult and adult mouse heart tissue's regenerative ability is minimal. But inhibition or inactivation of the Hippo signaling pathway and overexpression of YAP could improve cardiomyocytes' regenerative ability to a certain extent. Conversely, the specific deletion of YAP will significantly impair the regeneration ability of the neonatal mouse heart.

In short, the Hippo signaling pathway has a regulatory effect on the regeneration process of various tissues. In addition, since cell biology and biomaterial technology are the basis of cell tissue regeneration therapy, biomaterials may play a vital role in affecting cell recoding and fate by triggering gene expression. Understanding the interaction between cells and biomaterials may provide a theoretical basis for biomaterials designed for clinical applications in tissue regeneration.

\section{The Hippo signaling pathway involved in regulating the self-renewal of stem cells}

Cancer stem cells have the potential for self-renewal and transformation, which is the main reason for patients' poor prognosis. The Hippo signaling pathway is involved in regulating normal organ development and progenitor cell differentiation [128]. YAP/TAZ is highly expressed in stem cells of various tissues, suggesting that YAP/TAZ is involved in stem cell self-renewal and conversion. Overexpression of YAP or the inactivation of upstream inhibitors of the Hippo pathway activates the expression of YAP. It is found that the progenitor cell expansion and differentiation ability are weakened, and the skin, intestine, and other tissues are abnormally proliferated. Five days after YAP overexpression, the differentiation ability of intestinal stem cells was weakened, and the overexpression of YAP was stopped simultaneously. It was found that the differentiation ability of intestinal stem cells was restored.

Similarly, the phenotype of MST1/2 knockout in mice is the same as that of YAP overexpression, which indicates that YAP is participated the regulation of intestinal stem cell self-renewal and differentiation. In the early development of the skin, YAP is distributed in the nucleus of a single layer of skin cells as the skin begins to stratify. YAP translocates to basal cells with strong proliferation ability, and the expression activity decreases with cell proliferation. When YAP is overexpressed in skin progenitor cells, the skin tissue layer becomes thicker, and basal cells increase. At the same time, the cells that could proliferate are no longer limited to the basal layer. In addition, the ability of progenitor cell clone formation is enhanced, suggesting that stem cells have enhanced self-renewal. When specifically knocking out YAP or interfering with the expression of TEAD, the skin tissue layer becomes thinner, and the formation of multiple skins such as limbs, eyes, mouth, and nose is hindered, and basal cells are reduced. These results suggest that the Hippo signaling pathway could promote the self-renewal of epithelial stem cells and inhibit terminal differentiation.

\section{Conclusions}

The Hippo signaling pathway regulates organ development, tissue regeneration, and stem cell self-renewal. It does not act solely but interacts with other signaling pathways. The Hippo signaling pathway is also complicated in the occurrence and development of various human cancers. The nuclear localization and overexpression of YAP/TAZ have been confirmed in many human cancers. However, the underlying mechanism of Hippo pathway 
dysregulation and YAP/TAZ activation is still not well understood. Much efforts are needed to continue to study the biological functions of the Hippo signaling pathway in-depth, which is beneficial to the future research and treatment of tissue regeneration, stem cell diseases, and cancers. Through regulating it, the specificity and targeting of tumor treatment will be significantly improved, and at the same time, the damage of normal stem cells by drug treatment is expected to be significantly decreased.

\section{Abbreviations}

MST1/2: mammalianSTE20-like protein kinase; SAV1: human Salvadorhomology 1; LATS1/2: large tumor suppressor1/2; MOB1: MOB kinase activator; YAP: Yes-associated protein; TAZ: Tafazzin; TEADs: TEA domain family members; NDR1/2: Late embryogenesis abundant (LEA) hydroxyproline-rich glycoprotein family; FOXO1: forkhead box O1; TJs: tight junctions (TJs); AJs: adhesive connections; DUBs: ubiquitin-specific protease family; USP9X: ubiquitin specific peptidase 9 X-linked; AMOT: angiomotin; FAT: FAT atypical cadherin 1; Ex: Expande; PCP: Planar Cell Polarity; GPCRs: G Protein-Coupled Receptors; PARs: G protein-coupled receptor-protease activated receptor; IGF-1: insulin like growth factor 1; PI3K: phosphatidylinositol 3-kinase; VEGF: vascular endothelial growth factor; AMPK: Protein kinase AMP-activated catalytic subunit alpha 1; AMP: adenine phosphoribosyltransferase; AMOTL1: angiomotin like 1; PGE2: prostaglandin 2; HR: hypoxia-reoxygenation; ATF4: activating transcription factor 4; NEDD4.2: E3 ubiquitin-protein ligase Nedd-4; WWP1: WW domain containing E3 ubiquitin protein ligase 1; FOXO1: forkhead box O1; SOD: superoxide dismutase 2; HBV: hepatitis B virus; $\mathrm{HCV}$ : hepatitis $\mathrm{C}$ virus; $\mathrm{MCV}$ : infectious mollusc virus; HPV: human papillomavirus; Mupy V: mouse polyomavirus; KSHV: Kaposi Sarcoma-associated herpesvirus; EBV: Barr virus; ZIKA: Zika virus; ERBB4: erb-b2 receptor tyrosine kinase 4; EGR-1: early growth response 1; TBX5: T-box transcription factor 5; EGF: epidermal growth factor; FGF: fibroblast growth factor; CTGF: Connective tissue growth factor; CCN1: cellular communication network factor 1; DLAP1: Drosophila melanogaster; PPAR: peroxisome proliferator activated receptor; MCK: creatine kinase; Src: non-receptor tyrosine kinase; STING: stimulator of interferon response cGAMP interactor 1; ECM: extracellular matrix; MyoD: myogenic differentiation factor 1; DIAP1: drosophila apoptosis inhibitor; ALK: anaplastic lymphoma kinase; BCL2: B cell leukemia/ lymphoma 2; TBK1: TANK-bindingkinase1; PCD: programmed cell death; AREG: amphiregulin; BMP4: bone morphogen-netic protein 4; FAK: focal adhesion kinase; ILK: integrin-linked kinase; AICAR: an AMP mimic; VP: Verteporfin; VGLL4: vestigial like family member 4; CDX2: caudal type homeobox 2; CA3: carbonic anhydrase 3; SETD7: SET domain containing 7; LPA: Lysophosphatidic acid; NF2: neurofibromin 2.

\section{Acknowledgements}

This work was supported in part by grants from the National Natural Science Foundation of China (82072374) and the program of China Scholarships Council (No. 202006090323).

\section{Authors' contributions}

M.W and M.D. write the manuscript. M.W and D.W. draw the figures. W.X., Z.Z. and C.G. review the manuscript. Z.Z. and C.G. also guide the writing ideas.

\section{Data availability} author.

All data can be obtained from the corresponding

\section{Competing Interests}

The authors have declared that no competing interest exists.

\section{References}

[1] Matallanas D, Romano D, Hamilton G, Kolch W, O'Neill E. A Hippo in the ointment: MST signalling beyond the fly. CELL CYCLE. 2008;7:879-84.

[2] Tsutomu, Oka, Virginia, Mazack, Marius, Sudol. Mst2 and Lats Kinases Regulate Apoptotic Function of Yes Kinase-associated Protein (YAP). J BIOL CHEM. 2008;283: 27534-27546.

[3] Cairns L, Patterson A, Weingartner KA, Koehler TJ, DeAngelis DR, Tripp KW, et al. Biophysical characterization of SARAH domain-mediated multimerization of Hippo pathway complexes in Drosophila. J BIOL CHEM. 2020;295: 6202-6213.

[4] Aharonov A, Shakked A, Umansky KB, Savidor A, Genzelinakh A, Kain D, et al. ERBB2 drives YAP activation and EMT-like processes during cardiac regeneration. NAT CELL BIOL. 2020;22:1346-56.

[5] Luo, Xuelian, Hara, Mayuko, Pan, Duojia, et al. Structural basis for Mob1dependent activation of the core Mst-Lats kinase cascade in Hippo signaling. Genes and Development: a Journal Devoted to the Molecular Analysis of Gene Expression in Eukaryotes, Prokaryotes, and Viruses. 2015; 29: 1416-1431.

[6] Robinson BS, Moberg KH. Drosophila endocytic neoplastic tumor suppressor genes regulate Sav/Wts/Hpo signaling and the c-Jun N-terminal kinase pathway. CELL CYCLE. 2011; 10:4110-8.

[7] Swaroop B, Kanumuri R, Ezhil I, Sampangi J, Kremerskothen J, Rayala SK, et al. KIBRA connects Hippo signaling and cancer. EXP CELL RES. 2021; 403: 112613.

[8] Basu S, Totty NF, Irwin MS, Sudol M, Downward J. Akt phosphorylates the Yes-associated protein, YAP, to induce interaction with 14-3-3 and attenuation of p73-mediated apoptosis. MOL CELL. 2003; 11:11-23.

[9] Hoffken V, Hermann A, Pavenstadt H, Kremerskothen J. WWC Proteins: Important Regulators of Hippo Signaling in Cancer. CANCERS. 2021;13: 306.

[10] D. K, Ramireddy S, P. R, C. S. Expediting dynamics approach to understand the influence of 14-3-3 $\zeta$ causing metastatic cancer through the interaction of YAP1 and B -TRCP. MOL BIOSYST. 2017; 13:1981-92.

[11] Mohajan S, Jaiswal PK, Vatanmakarian M, Yousefi H, Sankaralingam S, Alahari SK, et al. Hippo pathway: Regulation, deregulation and potential therapeutic targets in cancer. CANCER LETT. 2021; 507:112-23.

[12] Cho YS, Li S, Wang X, Zhu J, Zhuo S, Han Y, et al. CDK7 regulates organ size and tumor growth by safeguarding the Hippo pathway effector Yki/Yap/Taz in the nucleus. GENE DEV. 2020;34:53-71.

[13] Kang W, Huang T, Zhou Y, Zhang J, Lung RWM, Tong JHM, et al. miR-375 is involved in Hippo pathway by targeting YAP1/TEAD4-CTGF axis in gastric carcinogenesis. Cell Death and Disease. 2018; 9: 92

[14] Niu K, Liu YL, Zhou ZJ, Wu XF, Wang HW, Yan JZ. Antitumor Effects of Paeoniflorin on Hippo Signaling Pathway in Gastric Cancer Cells. J ONCOL. 2021; 2021: 4724938. 
[15] Nicolay BN, Bayarmagnai B, Islam A, López Bigas N, Frolov MV. Cooperation between $\mathrm{dE} 2 \mathrm{~F} 1$ and $\mathrm{Yki} / \mathrm{Sd}$ defines a distinct transcriptional program necessary to bypass cell cycle exit. Genes Development. 2011;25: 323-35.

[16] Chen Y, Han H, Seo G, Vargas RE, Wang W. Systematic analysis of the Hippo pathway organization and oncogenic alteration in evolution. SCI REP-UK. 2020;10:3173.

[17] Gundogdu, Hergovich. MOB (Mps one Binder) Proteins in the Hippo Pathway and Cancer. CELLS-BASEL. 2019; 8:569.

[18] Ren F, Zhang L, Jiang J. Hippo signaling regulates Yorkie nuclear localization and activity through 14-3-3 dependent and independent mechanisms. DEV BIOL. 2010;337: 303-12

[19]. Zhang L, Ren F, Zhang Q, Chen Y, Wang B, Jiang J. The TEAD/TEF Family of Transcription Factor Scalloped Mediates Hippo Signaling in Organ Size Control. DEV CELL. 2008;14:377-87.

[20] Emami SS, Zhang D, Yang X. Interaction of the Hippo Pathway and Phosphatases in Tumorigenesis. CANCERS. 2020;12:2438.

[21] Meng Z, Moroishi T, Guan KL. Mechanisms of Hippo pathway regulation. Genes Dev. 2016;30:1-17.

[22] Dasgupta I, Mccollum D. Control of cellular responses to mechanical cues through YAP/TAZ regulation. J BIOL CHEM. 2019;294: 17693-17706.

[23] Qing-Hua, Yu, Qian, Yang. Diversity of tight junctions (TJs) between gastrointestinal epithelial cells and their function in maintaining the mucosal barrier. CELL BIOL INT. 2009;33:78-82.

[24] Zhao H, Liang Y, Xu Z, Wang L, Zhou F, Li Z, et al. N-glycosylation affects the adhesive function of E-Cadherin through modifying the composition of adherens junctions (AJs) in human breast carcinoma cell line MDA-MB-435. J CELL BIOCHEM. 2010; 104:162-75.

[25] Wang Y, Li J, Gao Y, Luo Y, Xiao ZXJ. Hippo kinases regulate cell junctions to inhibit tumor metastasis in response to oxidative stress. REDOX BIOL. 2019; 26:101233.

[26] Li L, Dong L, Hui J, Gao F, Yan J,Zhonghua W.et al. Effects of Hippo signaling on anti-oxidative stress of mesenchymal stem cells in vitro. Chinese Critical Care Medicine. 2018;30:296-301.

[27] Mana-Capelli S, Mccollum D. Angiomotins stimulate LATS kinase autophosphorylation and act as scaffolds that promote Hippo signaling. J BIOL CHEM. 2018;293:18230-41.

[28] Mussell A, Frangou C, Zhang J. Regulation of the Hippo signaling pathway by deubiquitinating enzymes in cancer. Genes Diseases. 2019;6:335-41.

[29] C., Ling, Y., Zheng, F., Yin, et al. The apical transmembrane protein Crumbs functions as a tumor suppressor that regulates Hippo signaling by binding to Expanded. Proceedings of the National Academy of Sciences. 2010:107:10532-7.

[30] Robinson BS, Huang J, Hong Y, Moberg KH. Crumbs Regulates Salvador/ Warts/Hippo Signaling in Drosophila via the FERM-Domain Protein Expanded. CURR BIOL. 2010;20:582-90.

[31] Schimizzi GV, Maher MT, Loza AJ, Longmore GD. Disruption of the Cdc42/Par6/aPKC or Dlg/Scrib/Lgl Polarity Complex Promotes Epithelial Proliferation via Overlapping Mechanisms. PLOS ONE. 2016;11:e159881.

[32] Lyczak R, Gomes JE, Bowerman B. Heads or tails: cell polarity and axis formation in the early Caenorhabditis elegans embryo. DEV CELL. 2002;3:157-66

[33] Elizabeth, Silva, And, Yonit, Tsatskis, And, et al. The Tumor-Suppressor Gene fat Controls Tissue Growth Upstream of Expanded in the Hippo Signaling Pathway. CURR BIOL. 2006;16:2081-9.

[34] Shen HY, Huang CZ, Wu JY, Li J, Hu T, Wang ZL, et al. SCRIB Promotes Proliferation and Metastasis by Targeting Hippo/YAP Signalling in Colorectal Cancer. FRONTIERS IN CELL AND DEVELOPMENTAL BIOLOGY. 2021; 9:656359.

[35] Huang Y, Pan M, Shu H, He B, Zhang F, Sun L. Vascular endothelial growth factor enhances tendon-bone healing by activating Yes-associated protein for angiogenesis induction and rotator cuff reconstruction in rats. J CELL BIOCHEM. 2020; 121:2343-53

[36] Silva E, Tsatskis Y, Gardano L, Tapon N, McNeill H. The Tumor-Suppressor Gene fat Controls Tissue Growth Upstream of Expanded in the Hippo Signaling Pathway. CURR BIOL. 2006; 16:2081-9.

[37] Chen Z, Chu S, Wang X, Sun Y, Xu T, Mao Y, et al. MiR-16a Regulates Milk Fat Metabolism by Targeting Large Tumor Suppressor Kinase 1 (LATS1) in Bovine Mammary Epithelial Cells. J AGR FOOD CHEM. 2019; 67:11167-78.

[38] Rauskolb C, Pan G, Reddy BVVG, Oh H, Irvine KD. Zyxin Links Fat Signaling to the Hippo Pathway. PLOS BIOL. 2011; 9: e1000624.

[39] Li KJ, Wu CH, Shen CY, Kuo YM, Yu CL, Hsieh SC. Membrane Transfer from Mononuclear Cells to Polymorphonuclear Neutrophils Transduces Cell Survival and Activation Signals in the Recipient Cells via Anti-Extrinsic Apoptotic and MAP Kinase Signaling Pathways. PLOS ONE. 2016;11: e156262.

[40] Jalabert A, Vial G, Guay C, Wiklander OPB, Nordin JZ, Aswad H, et al. Exosome-like vesicles released from lipid-induced insulin-resistant muscles modulate gene expression and proliferation of beta recipient cells in mice. DIABETOLOGIA. 2016; 59:1049-58

[41] Ying J, Wang $\mathrm{P}$, Zhang $\mathrm{S}$, Xu T, Zhang L, Dong R, et al. Transforming growth factor-beta1 promotes articular cartilage repair through canonical Smad and Hippo pathways in bone mesenchymal stem cells. LIFE SCI. 2018;192:84-90.

[42] Hui, Cai, Yan, Xu. The role of LPA and YAP signaling in long-term migration of human ovarian cancer cells. Cell Communication \& Signaling. 2013;11:31.
[43] Basu-Roy U, Han E, Rattanakorn K, Gadi A, Mansukhani A. PPAR $\gamma$ agonists promote differentiation of cancer stem cells by restraining YAP transcriptional activity. Oncotarget. 2016;7:60954-70.

[44] Qin D, Zhu J, Ji C, et al. Monoclonal antibody to six transmembrane epithelial antigen of prostate-4 influences insulin sensitivity by attenuating phosphorylation of P13K (P85) and Akt: Possible mitochondrial mechanism. J BIOENERG BIOMEMBR. 2011;43: 247-55.

[45] Cobbaut M, Karagil S, Bruno L, de la Loza MDCD, Elbediwy A. Dysfunctional Mechanotransduction through the YAP/TAZ/Hippo Pathway as a Feature of Chronic Disease. CELLS-BASEL. 2020;9:151.

[46] Borreguero-Muñoz N, Fletcher GC, Aguilar-Aragon M, Elbediwy A, VincentMistiaen ZI, Thompson BJ. The Hippo pathway integrates PI3K - Akt signals with mechanical and polarity cues to control tissue growth. PLOS BIOL. 2019;17: e3000509.

[47] Ying L, Jinming W, Weiliang Z. Regulation and mechanism of YAP/TAZ in the mechanical microenvironment of stem cells. Molecular Medicine Reports. 2021;24:506.

[48] Seo J, Kim J. Regulation of Hippo signaling by actin remodeling. BMB REP. 2018;51: 151-6.

[49] Dobrokhotov O, Samsonov M, Sokabe M, Hirata H. Mechanoregulation and pathology of YAP/TAZ via Hippo and non-Hippo mechanisms. Clinical and Translational Medicine. 2018;7: e23.

[50] Sun M, Spill F, Zaman MH. A Computational Model of YAP/TAZ Mechanosensing. BIOPHYS J. 2016;110:2540-50.

[51] Lieberthal W, Zhang L, Patel VA, Levine JS. AMPK protects proximal tubular cells from stress-induced apoptosis by an ATP-independent mechanism: potential role of Akt activation. American Journal of Physiology Renal Physiology. 2011;301:F1177.

[52] Contreras-Lopez R, Elizondo-Vega R, Luque-Campos N, Torres MJ, Pradenas C, Tejedor G, et al. The ATP synthase inhibition induces an AMPK-dependent glycolytic switch of mesenchymal stem cells that enhances their immunotherapeutic potential. THERANOSTICS. 2021;11:445-60.

[53] Zhengdong J, Xin C, Ke C, Liankang S, Luping G, Cancan Z, et al. YAP Inhibition by Resveratrol via Activation of AMPK Enhances the Sensitivity of Pancreatic Cancer Cells to Gemcitabine. NUTRIENTS. 2016;8:546.

[54] Jiang J, Chen S, Li K, Zhang C, Tan Y, Deng Q, et al. Targeting autophagy enhances heat stress-induced apoptosis via the ATP-AMPK-mTOR axis for hepatocellular carcinoma. Int J Hyperthermia. 2019;36:499-510.

[55] Liu Y, Ren H, Zhou Y, Shang L, Shi X. The hypoxia conditioned mesenchymal stem cells promote hepatocellular carcinoma progression through YAP mediated lipogenesis reprogramming. J EXP CLIN CANC RES. 2019;38:228.

[56] Geng C, Wei J, Wu C. Yap-Hippo pathway regulates cerebral hypoxiareoxygenation injury in neuroblastoma N2a cells via inhibiting ROCK1/ F-actin/mitochondrial fission pathways. ACTA NEUROL BELG. 2020;120:879-92.

[57] Rajesh K, Krishnamoorthy J, Gupta J, Kazimierczak U, Koromilas AE. The eIF2a serine 51 phosphorylation-ATF4 arm promotes HIPPO signaling and cell death under oxidative stress. Oncotarget. 2016;7:51044-58.

[58] Erbao, Zhang, Xuezhi, He, Chongguo, Jun, et al. A novel long noncoding RNA HOXC-AS3 mediates tumorigenesis of gastric cancer by binding to YBX1. GENOME BIOL. 2018;19:154.

[59] Dahlgren L, Dahlstrand H, Lindquist D, Högmo A, Björnestål L, Lindholm J, et al. Human papillomavirus is more common in base of tongue than in mobile tongue cancer and is a favorable prognostic factor in base of tongue cancer patients. INT J CANCER. 2004;112:1015-9.

[60] Alzahrani F, Clattenburg L, Muruganandan S, Bullock M, MacIsaac K, Wigerius $\mathrm{M}$, et al. The Hippo component YAP localizes in the nucleus of human papilloma virus positive oropharyngeal squamous cell carcinoma. Journal of Otolaryngology - Head \& Neck Surgery. 2017;46:15

[61] Xufeng L, Rui Z, Mengji L, Shi L, Hideo AB, Guido G, et al. Hippo Pathway Counter-Regulates Innate Immunity in Hepatitis B Virus Infection. FRONT IMMUNOL. 2021: $12: 684424$

[62] Mohammad O. E. A, Ubai K. A, Maram A. S, Rawya A. A, Mahmoud K, Ghimja F, et al. EBV Associated Breast Cancer Whole Methylome Analysis Reveals Viral and Developmental Enriched Pathways. FRONT IMMUNOL. 2018; 8:316.

[63] Wang Z, Lu W, Zhang Y, Zou F, Jin Z, Zhao T. The Hippo Pathway and Viral Infections. FRONT MICROBIOL. 2020;10: 3033.

[64] Min-Kyu K, Ju-Won J, Suk-Chul B. DNA binding partners of YAP/TAZ. BMB REP. 2018;51:126-33.

[65] Benedetto AD, Mottolese M, Sperati F, Ercolani C, Maugeri-Saccà M. The Hippo transducers TAZ/YAP and their target CTGF in male breast cancer. Oncotarget. 2016;7:43188-98.

[66] D., Lai, K., C., Ho, Y., et al. Taxol Resistance in Breast Cancer Cells Is Mediated by the Hippo Pathway Component TAZ and its Downstream Transcriptional Targets Cyr61 and CTGF. CANCER RES. 2011;71:2728-38.

[67] Shimomura T, Miyamura N, Hata S, Miura R, Hirayama J, Nishina H. The PDZ-binding motif of Yes-associated protein is required for its co-activation of TEAD-mediated CTGF transcription and oncogenic cell transforming activity. Biochemical \& Biophysical Research Communications. 2014;443:917-23.

[68] Elaimy AL, Mercurio AM. Convergence of VEGF and YAP/TAZ signaling: Implications for angiogenesis and cancer biology. SCI SIGNAL 2018:11:u1165.

[69] Chen TH, Chen CY, Wen HC, Chang CC, Chang CH. YAP promotes myogenic differentiation via the MEK5-ERK5 pathway. Faseb Journal Official 
Publication of the Federation of American Societies for Experimental Biology. 2017;31:2963-72.

[70] Judson RN, Gray SR, Walker C, Carroll AM, Itzstein C, Lionikas A, et al. Constitutive Expression of Yes-Associated Protein (Yap) in Adult Skeletal Muscle Fibres Induces Muscle Atrophy and Myopathy. PLOS ONE. 2013;8:e59622.

[71] Won GW, Sung M, Lee Y, Lee YH. MST2 kinase regulates osteoblast differentiation by phosphorylating and inhibiting Runx2 in C2C12 cells. BIOCHEM BIOPH RES CO. 2019;512: 591-7.

[72] Apoorva V, Fu L, Chong TY, Nur HM, Chacko J, Hao F, et al. Biophysical studies and modelling indicate the binding preference of TAZ WW domain for LATS1 PPxY motif. Biochemical \& Biophysical Research Communications. 2018; 502:307-12

[73] M. RR, Gbur SC, Beitel GJ, Helen WC. Non-Canonical Roles for Yorkie and Drosophila Inhibitor of Apoptosis 1 in Epithelial Tube Size Control. PLOS ONE. 2014; 9:e101609.

[74] Gerlach SU, Sander M, Song S, Herranz H. The miRNA bantam regulates growth and tumorigenesis by repressing the cell cycle regulator tribbles. Life ence Alliance. 2019;2: e201900381.

[75] Lei D, Chengcheng L, Xuan Q, Yibing C, Lei W, Hao Y, et al. Quercetin inhibited mesangial cell proliferation of early diabetic nephropathy through the Hippo pathway. PHARMACOL RES. 2019;146: 104320.

[76] Becker H, Renner S, Technau GM, Berger C. Cell-Autonomous and Non-cellautonomous Function of Hox Genes Specify Segmental Neuroblast Identity in the Gnathal Region of the Embryonic CNS in Drosophila. PLOS GENET. 2016;12: e1005961.

[77] Zhang Q, Meng F, Chen S, Plouffe SW, Wu S, Liu S, et al. Hippo signalling governs cytosolic nucleic acid sensing through YAP/TAZ-mediated TBK1 blockade. NAT CELL BIOL. 2017;19: 362-74.

[78] Booth LA, Roberts JL, Dent P. The role of cell signaling in the crosstalk between autophagy and apoptosis in the regulation of tumor cell survival in response to sorafenib and neratinib. SEMIN CANCER BIOL. 2020;66:129-39.

[79] Tsuneki M, Madri JA. CD44 Influences Fibroblast Behaviors Via Modulation of Cell - Cell and Cell - Matrix Interactions, affecting Survivin and Hippo Pathways. J CELL PHYSIOL. 2016;231: 731-43.

[80] Pu N, Gao S, Yin H, Li J, Wu W, Fang Y, et al. Cell-intrinsic PD-1 promotes proliferation in pancreatic cancer by targeting CYR61/CTGF via the hippo pathway. CANCER LETT. 2019;460: 42-53.

[81] Sayedyahossein S, Li Z, Hedman AC, Morgan CJ, Sacks DB. IQGAP1 Binds to Yes-associated Protein (YAP) and Modulates its Transcriptional Activity. J BIOL CHEM. 2016; 291: 19261-73.

[82] Yang, Zhongzhou, Ji, Hongbin, Ren, Yan, et al. YAP Promotes Malignant Progression of Lkb1-Deficient Lung Adenocarcinoma through Downstream Regulation of Survivin. Cancer Research the Official Organ of the American Association for Cancer Research Inc. 2015; 75:4450-7.

[83] Salmon H, Franciszkiewicz K, Damotte D, Dieu-Nosjean M, Validire P, Trautmann A, et al. Matrix architecture defines the preferential localization and migration of $\mathrm{T}$ cells into the stroma of human lung tumors. The Journal of Clinical Investigation. 2012;122: 899-910.

[84] Marcus J, Bejerano-Sagie M, Patterson N, Bagchi S, Verkhusha VV, Connolly D, et al. Septin 9 isoforms promote tumorigenesis in mammary epithelial cells by increasing migration and ECM degradation through metalloproteinase secretion at focal adhesions. ONCOGENE. 2019; 38:5839-59.

[85] Hiemer SE, Zhang L, Kartha VK, Packer TS, Almershed M, Noonan V, et al. A YAP/TAZ-Regulated Molecular Signature is associated with Oral Squamous Cell Carcinoma. Molecular cancer research : MCR. 2015;13:957-68.

[86] Zhao Z, Zheng N, Wang L, Hou Y, Zhou X. Rottlerin exhibits antitumor activity via down-regulation of TAZ in non-small cell lung cancer. Oncotarget. 2017;8:7827.

[87] Hwang S, Jin M, Shin YH, Ki Choi S, Namkoong E, Kim M, et al. Role of LPA and the Hippo pathway on apoptosis in salivary gland epithelial cells. Experimental \& Molecular Medicine. 2014;46:e125.

[88] Lai D, Yang X. BMP4 is a novel transcriptional target and mediator of mammary cell migration downstream of the Hippo pathway component TAZ. CELL SIGNAL. 2013:25:1720-8.

[89] Qu J, Zhao H, Li Q, Pan P, Ma K, Liu X, et al. MST1 Suppression Reduces Early Brain Injury by Inhibiting the NF- $\kappa$ B/MMP-9 Pathway after Subarachnoid Hemorrhage in Mice. BEHAV NEUROL. 2018;2018:6470957.

[90] Zhang J, Ji J, Yu M, Overholtzer M, Smolen GA, Wang R, et al. YAP-dependent induction of amphiregulin identifies a non-cell-autonomous component of the Hippo pathway. NAT CELL BIOL. 2009;11:1444-50.

[91] Shih CT, Shiau CW, Chen YL, Chen LJ, Chao TI, Wang CY, et al. TD-92, a novel erlotinib derivative, depletes tumor-associated macrophages in non-small cell lung cancer via down-regulation of CSF-1R and enhances the anti-tumor effects of anti-PD-1. CANCER LETT. 2021:498:142-51.

[92] He C, Mao D, Hua G, Lv X, Chen X, Angeletti PC, et al. The Hippo/YAP pathway interacts with EGFR signaling and HPV oncoproteins to regulate cervical cancer progression. EMBO MOL MED. 2015;7:1426-49.

[93] Wong KF, Liu AM, Hong W, Xu Z, Luk JM. Integrin a 2 \& 1 inhibits MST1 kinase phosphorylation and activates Yes-associated protein oncogenic signaling in hepatocellular carcinoma. Oncotarget. 2016; 7: 77683-77695.

[94] Chakraborty S, Njah K, Pobbati AV, Lim YB, Raju A, Lakshmanan M, et al. Agrin as a Mechanotransduction Signal Regulating YAP through the Hippo Pathway. CELL REP. 2017;18: 2464-79.
[95] Wei SC, Fattet L, Tsai JH, Guo Y, Pai VH, Majeski HE, et al. Matrix stiffness drives epithelial - mesenchymal transition and tumour metastasis through a TWIST1 - G3BP2 mechanotransduction pathway. NAT CELL BIOL. 2015;17:678-88.

[96] Cosset É, Ilmjärv S, Dutoit V, Elliott K, von Schalscha T, Camargo MF, et al. Glut3 Addiction Is a Druggable Vulnerability for a Molecularly Defined Subpopulation of Glioblastoma. CANCER CELL. 2017;32:856-68.

[97] Dupont S, Morsut L, Aragona M, Enzo E, Giulitti S, Cordenonsi M, et al. Role of YAP/TAZ in mechanotransduction. NATURE. 2011;474:179-83.

[98] Sansores-Garcia L, Bossuyt W, Wada K, Yonemura S, Tao C, Sasaki H, et al. Modulating F-actin organization induces organ growth by affecting the Hippo pathway. EMBO J. 2011;30:2325-35.

[99] Wada K, Itoga K, Okano T, Yonemura S, Sasaki H. Hippo pathway regulation by cell morphology and stress fibers. DEVELOPMENT. 2011;138:3907.

[100]DeRan M, Yang J, Shen C, Peters EC, Fitamant J, Chan P, et al. Energy Stress Regulates Hippo-YAP Signaling Involving AMPK-Mediated Regulation of Angiomotin-like 1 Protein. CELL REP. 2014:9:495-503.

[101]Wang W, Xiao ZD, Li X, Aziz KE, Gan B, Johnson RL, et al. AMPK modulates Hippo pathway activity to regulate energy homeostasis. NAT CELL BIOL. 2015;17:490-9.

[102]Mo JS, Meng Z, Kim YC, Park HW, Hansen CG, Kim S, et al. Cellular energy stress induces AMPK-mediated regulation of YAP and the Hippo pathway. NAT CELL BIOL. 2015;17:500-10.

[103]Gibault F, Sturbaut M, Bailly F, Melnyk P, Cotelle P. Targeting Transcriptional Enhanced Associate Domains (TEADs). J MED CHEM. 2018;61:5057-72.

[104]Zeng RY, Dong JX. The Hippo Signaling Pathway in Drug Resistance in Cancer. CANCERS. 2021;13: 318.

[105]Coffey K. Targeting the Hippo Pathway in Prostate Cancer: What's New? CANCERS. 2021;13: 611

[106]Gibault F, Bailly F, Corvaisier M, Coevoet M, Huet G, Melnyk P, et al. Molecular Features of the YAP Inhibitor Verteporfin: Synthesis of Hexasubstituted Dipyrrins as Potential Inhibitors of YAP/TAZ, the Downstream Effectors of the Hippo Pathway. CHEMMEDCHEM. 2017;12:954-61.

[107]Wei H, Wang F, Wang Y, Li T, Xiu P, Zhong J, et al. Verteporfin suppresses cell survival, angiogenesis and vasculogenic mimicry of pancreatic ductal adenocarcinoma via disrupting the YAP-TEAD complex. CANCER SCI. 2017;108:478-87.

[108]Zhang Z, Lin Z, Zhou Z, Shen HC, Yan SF, Mayweg AV, et al. Structure-Based Design and Synthesis of Potent Cyclic Peptides Inhibiting the YAP-TEAD Protein-Protein Interaction. ACS MED CHEM LETT. 2014:5:993-8.

[109]Jiao S, Wang H, Shi Z, Dong A, Zhang W, Song X, et al. A Peptide Mimicking VGLL4 Function Acts as a YAP Antagonist Therapy against Gastric Cancer. CANCER CELL. 2014; 25:166-80.

[110]Jiao S, Li C, Hao Q, Miao H, Zhang L, Li L, et al. VGLL4 targets a TCF4 TEAD4 complex to coregulate Wnt and Hippo signalling in colorectal cancer. NAT COMMUN. 2017; 8:14058

[111]Song, Shumei, Xie, Min, Scott, Ailing, et al. A Novel YAP1 Inhibitor Targets CSC-Enriched Radiation-Resistant Cells and Exerts Strong Antitumor Activity in Esophageal Adenocarcinoma. MOL CANCER THER. 2018; 17:443-54.

[112] Barsyte-Lovejoy D, Li F, Oudhoff MJ, Tatlock JH, Dong A, Zeng H, et al. (R)PFI-2 is a potent and selective inhibitor of SETD7 methyltransferase activity in cells. Proceedings of the National Academy of Sciences. 2014;111:12853.

[113]Yu J, Alharbi A, Shan H, Hao Y, Snetsinger B, Rauh MJ, et al. TAZ induces lung cancer stem cell properties and tumorigenesis by up-regulating ALDH1A1. Oncotarget. 2017; 8:38426-43.

[114]Guerrant W, Kota S, Troutman S, Mandati V, Fallahi M, StemmerRachamimov A, et al. YAP Mediates Tumorigenesis in Neurofibromatosis Type 2 by Promoting Cell Survival and Proliferation through a COX-2-EGFR Signaling Axis. CANCER RES. 2016; 76:3507-19.

[115]Ghiso E, Migliore C, Ciciriello V, Morando E, Petrelli A, Corso S, et al. YAPDependent AXL Overexpression Mediates Resistance to EGFR Inhibitors in NSCLC. NEOPLASIA. 2017;19: 1012-21.

[116]Xinyan, Ji, Lihua, Song, Li, Sheng, et al. Cyclopeptide RA-V Inhibits Organ Enlargement and Tumorigenesis Induced by YAP Activation. CANCERS. 2018;10:449.

[117]Lin L, Sabnis AJ, Chan E, Olivas V, Cade L, Pazarentzos E, et al. The Hippo effector YAP promotes resistance to RAF- and MEK-targeted cancer therapies. NAT GENET. 2015;47: 250-6.

[118]Eisinger-Mathason TSK, Mucaj V, Biju KM, Nakazawa MS, Gohil M, Cash TP, et al. Deregulation of the Hippo pathway in soft-tissue sarcoma promotes FOXM1 expression and tumorigenesis. Proceedings of the National Academy of ences of the United States of America. 2015;112:3402-11.

[119]Fisher ML, Kerr C, Adhikary G, Grun D, Xu W, Keillor JW, et al. Transglutaminase Interaction with \{alpha\}6/\{beta\}4-Integrin Stimulates YAP1-Dependent \{Delta\}Np63\{alpha\} Stabilization and Leads to Enhanced Cancer Stem Cell Survival and Tumor Formation. CANCER RES. 2016;76: $7265-76$.

[120]Liu CY, Pobbati AV, Huang Z, Cui L, Hong W. Transglutaminase 2 is a Direct Target Gene of YAP/TAZ-Letter. CANCER RES. 2017; 77:4734-5.

[121]Hwang S, Jin M, Shin YH, Ki Choi S, Namkoong E, Kim M, et al. Role of LPA and the Hippo pathway on apoptosis in salivary gland epithelial cells. Experimental \& Molecular Medicine. 2014;46: e125.

[122]Ho LTY, Skiba N, Ullmer C, Rao PV. Lysophosphatidic Acid Induces ECM Production via Activation of the Mechanosensitive YAP/TAZ Transcriptional 
Pathway in Trabecular Meshwork Cells. Investigative Ophthalmology \& Visual ence. 2018;59: 1969-84.

[123]Kakiuchi-Kiyota S, Schutten MM, Zhong Y, Crawford JJ, Dey A. Safety Considerations in the Development of Hippo Pathway Inhibitors in Cancers. Frontiers in Cell and Developmental Biology. 2019;7:156.

[124]Guo J, Zeng H, Chen Y. Emerging Nano Drug Delivery Systems Targeting Cancer-associated Fibroblasts for improved Antitumor Effect and Tumor Drug Penetration. MOL PHARMACEUT. 2020; 17:1028-48.

[125]Cho YS, Li S, Wang X, Zhu J, Zhuo S, Han Y, et al. CDK7 regulates organ size and tumor growth by safeguarding the Hippo pathway effector Yki/Yap/Taz in the nucleus. Genes\&Development. 2020; 34:53-71.

[126]Tharehalli U, Svinarenko M, Kraus JM, Kühlwein SD, Szekely R, Kiesle U, et al. YAP Activation Drives Liver Regeneration after Cholestatic Damage Induced by Rbpj Deletion. 2018; 19: 3801.

[127]Meyer K, Morales-Navarrete H, Seifert S, Wilsch-Braeuninger M, Dahmen U, Tanaka EM, et al. Bile canaliculi remodeling activates YAP via the actin cytoskeleton during liver regeneration. MOL SYST BIOL. 2020;16:e8985.

[128]Lange AW, Sridharan A, Xu Y, Stripp BR, Perl A, Whitsett JA. Hippo/Yap signaling controls epithelial progenitor cell proliferation and differentiation in the embryonic and adult lung. J MOL CELL BIOL. 2015; 7:35-47. 